\title{
Fomentar Atractivos Turísticos en Guayaquil
}

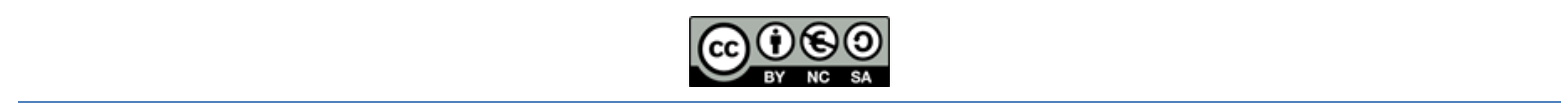

\section{Promote Tourist Attractions in Guayaquil}

Efraín Velasteguí López. ${ }^{1}$

Recibido: 16-09-2019 / Revisado: 18-09-2019 / Aceptado: 22-09-2019 / Publicado: 05-10-2019

Resumen.

DOI: https://doi.org/10.33262/concienciadigital.v2i4.934

Para fomentar y promocionar los sitios turísticos en el cantón La Maná perteneciente a la provincia de Cotopaxi, busca identificar los productos que brinda el sector para el desarrollo turístico del cantón y de la provincia para llegar a la elaboración de un plan de desarrollo turístico integral junto a los demás cantones de la provincia, previo a un diagnóstico de la infraestructura turística que brindan los atractivos situados en el cantón y las actividades que proponen para la distracción y esparcimiento de su población y los visitantes, además de, estudiar la posibilidad de nuevas alternativas de actividades y negocios innovadores orientados al turismo como una actividad económica alterna a la que el cantón posee por tradición la cual contribuiría al progreso económico general beneficiando de esta manera a la población que en el habita. A lo largo del desarrollo de la investigación se describe los pasos a seguir

Para el análisis del lugar, la población, productos que ofrece y los posibles productos que se puedan implementar según el avance de la propuesta lo cual nos conduce a obtener un trabajo integral el cual revele las maravillas que ofrece el cantó La Maná y lo que falta por ofrecer, así como lo que se debe mejorar para convertirse en uno de los destinos turísticos de mayor afluencia en el Ecuador.

Bajo este concepto, nace la idea de promover el desarrollo turístico de la ciudad de La Maná basada en la creación de micro negocios sustentables, que tengan un bajo impacto ambiental y no deterioren el entorno.

Palabras claves: Fomentar, promocionar, Sitios, Turísticos.

\footnotetext{
${ }^{1}$ Ciencia digital, Ambato, Ecuador, luisefrainvelastegui@cienciadigital.org
} 


\section{Abstract.}

To promote and promote tourist sites in the municipality of La Maná belonging to the province of Cotopaxi, it seeks to identify the products offered by the sector for the tourist development of the canton and the province to arrive at the elaboration of an integral tourist development plan together to the other cantons of the province, prior to a diagnosis of the tourism infrastructure provided by the attractions located in the canton and the activities they propose for the distraction and recreation of its population and visitors, in addition to studying the possibility of new alternatives of activities and innovative businesses oriented to tourism as an alternative economic activity to which the canton owns by tradition which would contribute to the general economic progress thus benefiting the population that inhabits it. Throughout the development of the investigation, the steps to be followed are described.

For the analysis of the place, the population, products offered and the possible products that can be implemented according to the progress of the proposal which leads us to obtain an integral work which reveals the wonders offered by the La Maná song and what is missing to offer, as well as what should be improved to become one of the tourist destinations of greater affluence in Ecuador.

Under this concept, the idea of promoting the tourist development of the city of La Maná based on the creation of sustainable micro businesses, which have a low environmental impact and do not deteriorate the environment is born.

Keywords: Encourage, promote, Sites, Tourist.

\section{Introducción.}

Hoy en día, es posible apreciar el dinamismo de ciertas economías basadas en el desarrollo de su potencial turístico, debido a que esta actividad genera un gran flujo de divisas alrededor del mundo y a su vez involucra a diversos sectores, entre los que se destacan empresas enfocadas en el hospedaje, alimentación, transportación, operación, entre otras.

La actividad productiva del turismo puede ser ampliamente beneficiosa, pero al mismo tiempo, podría ser considerado como destructivo si no es manejado adecuadamente y con responsabilidad, debido a que es posible afectar el entorno y por el contrario la saturación de turistas en un mismo sitio podría deteriorar la riqueza de los patrimonios naturales y culturales de cualquier país. Ante esta situación problemática, es que surgen diversas actividades y grupos económicos con gran interés en convertir las prácticas tradicionales de los sectores empresariales turísticos a manejarlos responsablemente mediante el empleo de prácticas de sostenibilidad. 
Las prácticas de sostenibilidad intentan modificar la forma de hacer negocios enfocadas en el "Desarrollo Sostenible"; es decir, "el desarrollo que satisface las necesidades del presente sin comprometer los recursos de las generaciones futuras para satisfacer sus propias necesidades.

Se pretende tener una actividad sostenible y sustentable cuyo impacto económico, social y ambiental permitan complacer las necesidades de los turistas y pobladores de la zona. Esta propuesta se trata de dar e inculcar ideas y opciones de negocios que se podría ubicar la parte que se estaría involucrada, siendo esto los pobladores de la zona y a la vez fomentar el crecimiento económico y turístico para que así mejore la calidad de vida, pero sin descuidar el recurso natural.

\section{Marco Teórico}

\section{Fomentar}

Según (Melgosa. F, 2004., Sánchez. M, 2008., Sudarsky. J, 2015)Afirma que: fomentar es el amplio interés que ha despertado el concepto de cultura ciudadana y su aplicación como política pública proviene, sin duda, de la experiencia de Bogotá a partir de 1995, pero también encuentra explicación en el nuevo énfasis que se ha puesto en medios académicos a la dimensión cultural del desarrollo económico, político y social.

Un ciudadano libre y responsable es, ante todo, alguien que actúa con plena conciencia y libre albedrío sobre la base de sus derechos y deberes. Su responsabilidad supone que tiene un papel que cumplir en la construcción de ciudadanía, en el cumplimiento de las normas, en la creación y consolidación de relaciones sociales basadas en la confianza, en la tolerancia y en el respeto por los demás.

Tales tareas no son competencia exclusiva del Estado. Aun en las sociedades más avanzadas, la capacidad del Estado para hacer cumplir las normas es limitada; por consiguiente, el ciudadano tiene una responsabilidad vital que cumplir, no sólo consigo mismo sino con los demás.

\section{Criterio Personal}

Éstas sólo son posibles con el concurso de la ciudadanía, que puede aprender a responsabilizarse de su propia seguridad y de la de los demás mediante la previsión, la minimización de los factores de riesgo y la colaboración con los otros ciudadanos y con las autoridades en la prevención de los delitos.

En el marco comunitario europeo también han sido muy diversas las medidas que directamente o indirectamente han tratado de fomentar el turismo en el ámbito de los estados miembros en la mismas o diferentes líneas de actuación autonómica. 
Para fomentar la diversificación del turismo, la desconcentración en el sol y la playa, la costa y el verano. Fomentar nuevos productos, nuevo destino de viajes fuera de temporada. Fomentar formas incipientes y alternativas de turismo: turismo rural, turismo activo, nuevas formas de turismo cultural, etc.

Figura N 1 Turismo
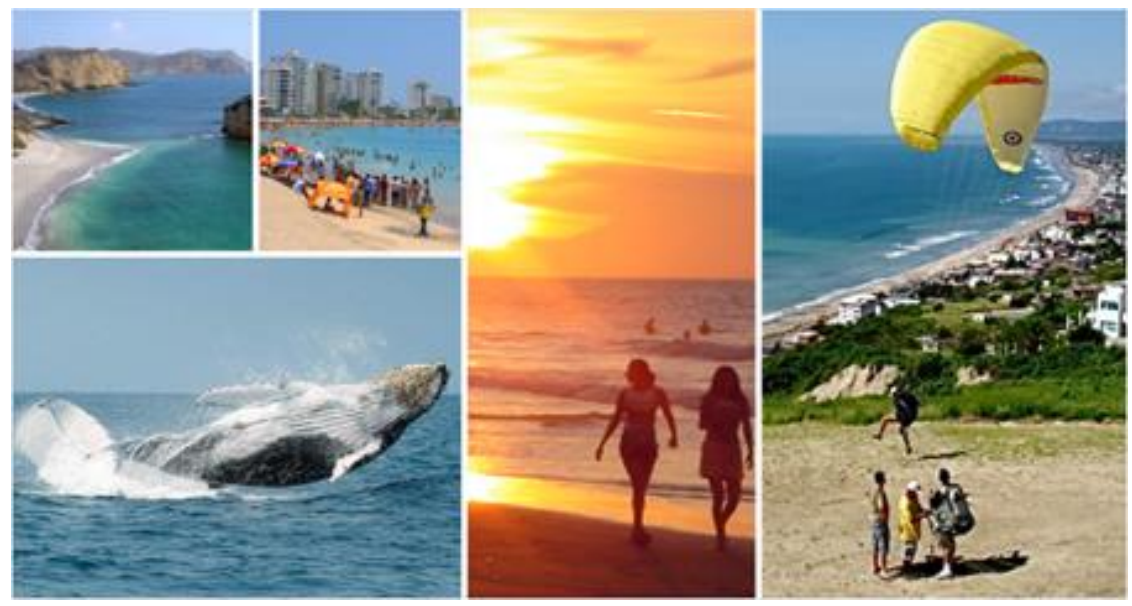

Fuente: https://ec.viajandox.com/costa

Según (ALMONTE. J, 2016., Granadinas. E, 2014., Guerrero. E, 2014) afirma que: El turismo es uno de los valores y un referente importante de nuestro país. México está decidido a impulsar y facilitar la productividad, porque al haber éxito en el sector, habrá gente entonces que visite el país, por conducto del turismo. Porque las economías en la medida que transitan hacia la modernidad han venido pasando las economías agrícolas, a economía industriales y luego a economía de servicio. El turismo cultural en la raya se sustenta en los numerosos recursos enmarcados dentro del apartado del patrimonio cultural material e inmaterial. la relación del inventario del patrimonio histórico-artístico es muy extensa.

\section{Criterio Personal}

Las primeras manifestaciones del Turismo la encontramos en los hechos que refieren viajes como antigua forma de turismo. En la literatura podemos observar claramente esos viajes: La Eneida, La Odisea y La Ilíada. Los Juegos Olímpicos Griegos causando muchos desplazamientos con motivaciones características de turismo y recreación. Edad Media: el deseo de conquista motiva desplazamientos humanos con deseos de conquistar. Guerra de la "Cruzadas", peregrinaciones a Santiago de Compostela, existencia de posadas en los caminos, expansión y búsqueda de nuevas rutas comerciales.

A mediados del siglo XVI aparece en Italia el uso de y carrozas, en Alemania surge el de uso ligero denominado Berlina. El siglo XVII se caracteriza por el del Caballero y la Diligencia. En el siglo XIX se desarrollan las líneas ferroviarias especialmente en Europa y Estados 
Unidos. A mediados de este siglo se considera a los Museos y Monumentos como lugares de interés turístico y se establecen regulaciones de horarios y precios. Surge la de por el señor Thomas Cook en 1841, quien ofrecía servicios de medidora entre la demanda del cliente y una oferta de transporte, alojamientos y atractivos Turísticos.

Según (Morante, L. 2016., Perez , J. 2017., Pozo, R. 2013) Afirma que: fomentar es el amplio interés que ha despertado el concepto de cultura ciudadana y su aplicación como política pública proviene, sin duda, de la experiencia de Bogotá a partir de 1995, pero también encuentra explicación en el nuevo énfasis que se ha puesto en medios académicos a la dimensión cultural del desarrollo económico, político y social.

Un ciudadano libre y responsable es, ante todo, alguien que actúa con plena conciencia y libre albedrío sobre la base de sus derechos y deberes. Su responsabilidad supone que tiene un papel que cumplir en la construcción de ciudadanía, en el cumplimiento de las normas, en la creación y consolidación de relaciones sociales basadas en la confianza, en la tolerancia y en el respeto por los demás.

Tales tareas no son competencia exclusiva del Estado. Aun en las sociedades más avanzadas, la capacidad del Estado para hacer cumplir las normas es limitada; por consiguiente, el ciudadano tiene una responsabilidad vital que cumplir, no sólo consigo mismo sino con los demás.

La corresponsabilidad y la ayuda mutua para que el ciudadano actúe en armonía con la ley, son parte de la definición de cultura ciudadana. También suele ser limitada la capacidad del Estado para garantizar la paz y la seguridad.

Éstas sólo son posibles con el concurso de la ciudadanía, que puede aprender a responsabilizarse de su propia seguridad y de la de los demás mediante la previsión, la minimización de los factores de riesgo y la colaboración con los otros ciudadanos y con las autoridades en la prevención de los delitos.

En el marco comunitario europeo también han sido muy diversas las medidas que directamente o indirectamente han tratado de fomentar el turismo en el ámbito de los estados miembros en la mismas o diferentes líneas de actuación autonómica. En todo caso estas medidas se atienen al principio de subsidiariedad y suponen acciones muy diversas que han sido adoptadas por las instituciones comunitarias a partir de la Decisión del Consejo del 13 de julio de 1992, que aprobó un Plan de acciones comunitarias en favor del turismo y que se tradujo en programas y ayudas concretas.

\section{Criterio Personal}

Desde años atrás y con la necesidad de mejorar o implementar lugares servicios que definitivamente serian importante y relevantes en el ámbito socio económico de cierta 
región o lugar en el que se fomentara y se dará a conocer los nuevos servicios requeridos mediante esa operación como nuestros autores mencionan en el ámbito turístico lo más importante es buscar nuevos atractivos k llamen la atención del turista sin ser los mismos atractivos ya cotidianos sin embargo dirigiéndonos en el Cantón la mana se recomienda impulsar catastros de atractivos turísticos que los tiene pero k no han sido aún explotados y fomentados de una manera correcta para atraer turista de la zona nacionales y por qué no extranjeros prestando servicios de calidad y creando paquetes turísticos que sean accesibles para la diferentes clases sociales y a la medida de la economía de cada persona.

Para fomentar la diversificación del turismo, la desconcentración en el sol y la playa, la costa y el verano. Fomentar nuevos productos, nuevo destino de viajes fuera de temporada. Fomentar formas incipientes y alternativas de turismo: turismo rural, turismo activo, nuevas formas de turismo cultural, etc.

Según (Valbuena, F. 2015., Vizuete, F. 2015., Sañudo, M. 2015., Pozo, R. 2013)

afirma que: El turismo es uno de los valores y un referente importante de nuestro país. México está decidido a impulsar y facilitar la productividad, porque al haber éxito en el sector, habrá gente entonces que visite el país, por conducto del turismo. Porque las economías en la medida que transitan hacia la modernidad han venido pasando las economías agrícolas, a economía industriales y luego a economía de servicio.

Las primeras manifestaciones del Turismo la encontramos en los hechos que refieren viajes como antigua forma de turismo. En la literatura podemos observar claramente esos viajes: La Eneida, La Odisea y La Ilíada. Los Juegos Olímpicos Griegos causando muchos desplazamientos con motivaciones características de turismo y recreación. Edad Media: el deseo de conquista motiva desplazamientos humanos con deseos de conquistar. Guerra de la "Cruzadas", peregrinaciones a Santiago de Compostela, existencia de posadas en los caminos, expansión y búsqueda de nuevas rutas comerciales.

\section{Criterio Personal}

¿El turismo hoy en día y desde los inicios de sus pasos se ha centrado en qué? En que las personas se movilicen de un lugar a otro al principio obviamente las personas hacían turismo sin saber lo k era hoy en día el turismo es una potencia económica si la llamamos así la razón es porque genera movimiento de dinero dichos procesos están relacionados con alojamiento ,alimentación, transportación, intermediación, operación, recreación elementos principales para realizar turismo y $\mathrm{k}$ genera esto que el turista consuma servicios y genera divisas en el cual los beneficiarios son los prestadores de servicios y los establecimientos $\mathrm{k}$ estas debidamente adecuados para que el turista se sienta cómodo y pueda realizar las actividades turísticas con normalidad. 
El turismo cultural en la raya se sustenta en los numerosos recursos enmarcados dentro del apartado del patrimonio cultural material e inmaterial. la relación del inventario del patrimonio histórico-artístico es muy extensa.

Figura N 2 Atractivos

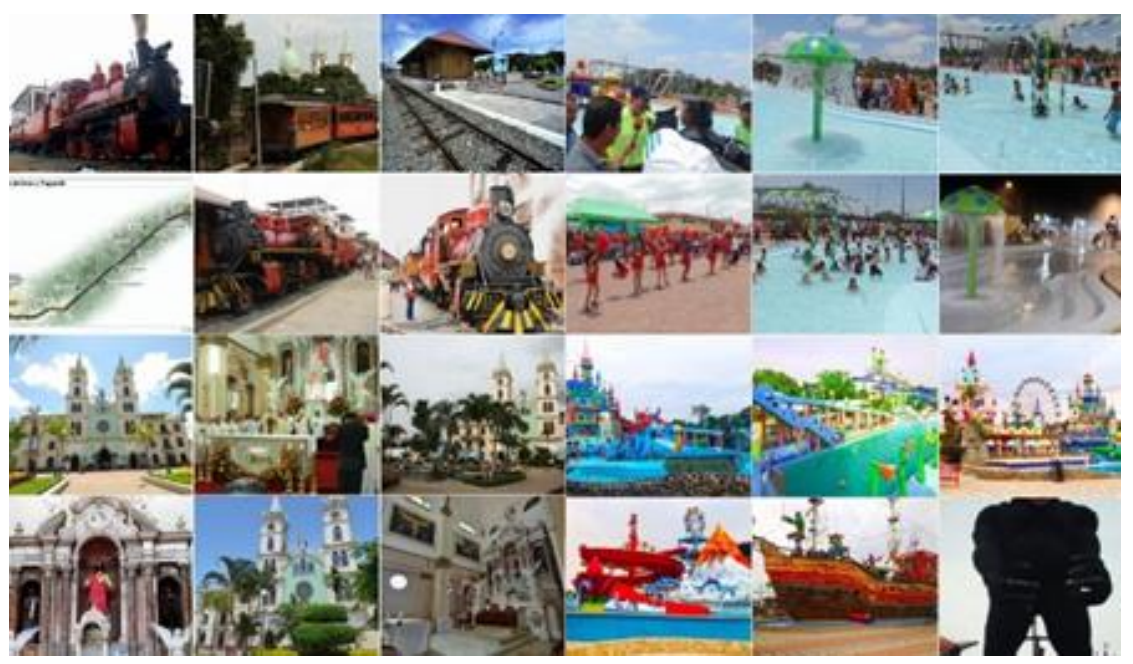

Fuente: http://municipiodeyaguachi.gob.ec/index.php/turismo/atractivos-

Según (Chávez. J, 2015., Guerrero. A. 2014., Lobato. J 2015., López. J 2016) Afirma que: Es un atractivo turístico es un sitio o un hecho que genera interés entre los viajeros. De este modo, puede tratarse de un motivo para que una persona tome la decisión de visitar una ciudad o un país.

El valor de los atractivos turísticos puede radicar en diferentes cuestiones. En algunos casos, se trata de espacios de importancia histórica. En otros, la atracción surge por la belleza natural. Más allá de lugares concretos (físicos), hay atractivos turísticos que son más bien simbólicos, como la gastronomía o la oferta de eventos.

El flujo turístico en el Ecuador se ha venido incrementando en los últimos años, teniendo un avance significativo en cifras de ingresos de divisas por esta actividad que beneficia nuestra economía. Desde el año 2002 hasta el año 2005 se ha incrementado el flujo de llegadas de extranjeros hacia nuestro país en un promedio de $8 \%$ anual, dejando en la economía local 486 millones de Dólares, cual evidencia que la situación socio política del Ecuador iba en vías de estabilización y permitía visitar nuestro país con cierta tranquilidad cual beneficiaba a la actividad turística, del año 2005 hacia el 2006, la llegada de turistas registrados en migración decayó en 1\%, siendo responsables de esta baja la mala situación política que nuestro país atravesaba, con el derrocamiento del ex - presidente Lucio Gutiérrez, problema que ocasionó inestabilidad política, económica y falta de garantías hacia extranjeros. 


\section{Criterio Personal}

Dentro de las exhibiciones estuvieron las pinturas de Ítalo Pilay, quien mostró uno de sus cuadros más representativos, como es al campesino tejiendo el sombrero de paja toquilla en honor a que en la parroquia Julcuy fue donde se tejió el primer sombrero.

"Con estos cuadros he recorrido varias ciudades de Ecuador y países como", dijo Pilay, y agregó que cuenta con unos 60 cuadros pintados y prepara otros. El seminario inició el martes y culminó ayer, y hubo alrededor de nueve expositores, entre nacionales y cubanos.

El turismo es uno de los valores y un referente importante de nuestro país. México está decidido a impulsar y facilitar la productividad, porque al haber éxito en el sector, habrá gente entonces que visite el país, por conducto del turismo.

Figura N 3 Ventas de extranjeros periodo 2010 - 2016 (millones)

$$
\begin{aligned}
& \text { VISITAS DE EXTRANJEROS } \\
& \text { PERIODO 2010-2016 (MILLONES) }
\end{aligned}
$$

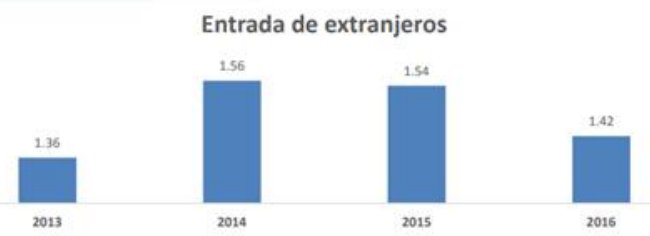

Fuente: INEC — Anuario de Entradas y Salidas internacionales 2016

\section{Análisis}

Porque las economías en la medida que transitan hacia la modernidad han venido pasando de economías agrícolas, a economías industriales y luego a economías de servicios, pero sabemos que en la medida que evolucionen más servicios financieros y turísticos, serán la clave y los motores de nuestra economía en el futuro; por eso, apostamos finalmente a tener un compromiso sólido con el estado de derecho trabajando e invirtiendo en paz en nuestro país".

Turismo es una actividad con gran potencial de crecimiento en el Guayas 
Figura N 4 Turismo en el Guayas

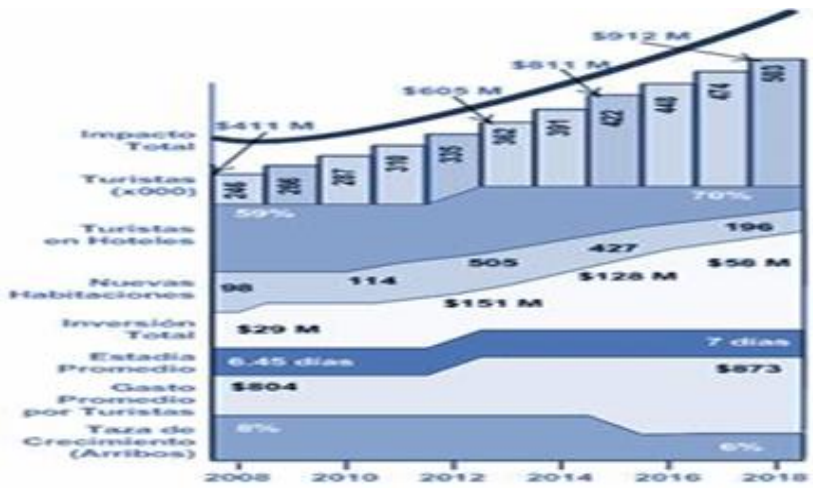

Fuente: https://www.suitesguayaquil.com/plan-maestro-turismo-guayas.html

\section{Análisis}

Porque las economías en la medida que transitan hacia la modernidad han venido pasando de economías agrícolas, a economías industriales y luego a economías de servicios, pero sabemos que en la medida que evolucionen más servicios financieros y turísticos, serán la clave y los motores de nuestra economía en el futuro; por eso, apostamos finalmente a tener un compromiso sólido con el estado de derecho trabajando e invirtiendo en paz en nuestro país".

\section{Conclusiones}

- La actividad turística peruana ha experimentado un constante crecimiento, tal como se ha podido observar a lo largo de estos últimos años; de ahí la necesidad de estar más preparados para enfrentar la demanda, cada vez más exigente y más cambiante.

- La utilización de la teoría denominada Competitividad Sistémica, ha permitido hacer un análisis y diagnóstico más profundo en relación al sector turismo en la Ciudad de Guayaquil; y es en base a esta metodología que se ha llegado a determinar los principales factores controlables y los no controlables.

- En la actualidad existen tres tipos de turismo que presentan una tendencia mundial de alto crecimiento; el ecoturismo, el turismo histórico - cultural y por último el turismo de aventura. Estos tipos de turismo representan nichos de mercado los cuales deben ser tomados en cuenta por toda estrategia turística. 
- Pero los más importante de todo en este plan de acción son los agentes involucrados en el sector. Sin concertación entre el sector público y privado la estrategia planteada no se puede concretar.

\section{Referencias bibliográficas.}

ALMONTE, J. (2016). TURISMO DE FRONTERA (III): PRODUCTOS TURISTICOS DE LA RAYA IBERICA. Europa: Servicio de Publicaciones de la Universidad de Huelva.

Chavez, J. (2015). El Diario. Recuperado el 24 de 10 de 2018, de http://www.eldiario.ec/noticias-manabi-ecuador/364511-buscan-fomentar-elturismo-local/

Granadinas, L. (2018). EcuRed. España.

Guerrero, E. (2014). Introducción al Turismo. Mexico : Patria S.A.

Guerrero , P. E. (2014). INTRODUCCIÓN AL TURISMO. Mexico: EBOOK. Recuperado $\begin{array}{lllllll}\text { el } & 24 & \text { de } & 10 & \text { de } & \text { de }\end{array}$ http://www.editorialpatria.com.mx/pdffiles/9786074384130.pdf

Lobato, J. (2015). turistica de La Maná. La Mana: Scrib.

Lopez, J. (2016). Ecuador busca la mejor estrategia para poder fomentar el turismo en los próximos años y aprovechar su potencial. Recuperado el 24 de 10 de 2018, de https://www.porconocer.com/ecuador/ecuador.html

Melgosa, F. J. (2004). Derecho y turismo. España: Universidad de Salamanca. Recuperado $\begin{array}{lllllll}\text { el } & 24 & \text { de } & 10 & \text { de } & 2018, & \text { de }\end{array}$ https://books.google.com.ec/books?id=mZIjPkOcAGAC\&pg=PA68\&dq=fomentar +en+el+ambito+turistico\&hl=es419\&sa $=$ X\&ved=0ahUKEwiY14H61 aneAhVCMn0KHTsMDmgQ6AEIJjAA\#v=o nepage $\& \mathrm{q}=$ fomentar\%20en $\% 20 \mathrm{el} \% 20$ ambito $\% 20$ turistico $\& \mathrm{f}=$ false 
Morante , L. (2016). Esquema material procesual de identidad. España

Perez , J. (2017). Definicion de actractivos turisticos . Mursia.

Pozo, R. (2013). Conceptos y principios de economía y metodologías utilizadas en la investigación económica. TENDENCIAS, 232.

Sañudo, M. (2015). DESARROLLO Prácticas y discursos emergentes en América Latina. $\begin{array}{lllllll}\text { Recuperado el } & 15 & \text { de } & 10 & \text { de }\end{array}$ http://biblioteca.clacso.edu.ar/clacso/gt/20160309021832/Desarrollo_practicas.pdf

Sánchez, M. (2008). Medida de la sostenibilidad turística: Propuesta de un índice sintético. España: Centro de Estudios Ramon Arces. Recuperado el 24 de 10 de 2018

Sudarsky, J. (2015). FOMENTAR LA CULTURA CIUDADANA. Recuperado el 24 de 10 de 2018, de https://www.mineducacion.gov.co/cvn/1665/articles95980_archivo_pdf24.pdf

Vizuete , F. (2015). La razón de ser de la Economía. Recuperado el 15 de 10 de 2018, de http://econopilar.com/wp-content/uploads/2015/09/Tema-01-La-economia-comociencia.pdf

Valbuena, F. (2015). TEORÍA SOBRE CAUSAS Y EFECTOS. Buenos Aires: Tratado de Filosofía. Recuperado el 23 de 10 de 2018, de http://www.fgbueno.es/edi/val/fvtgi35.pdf

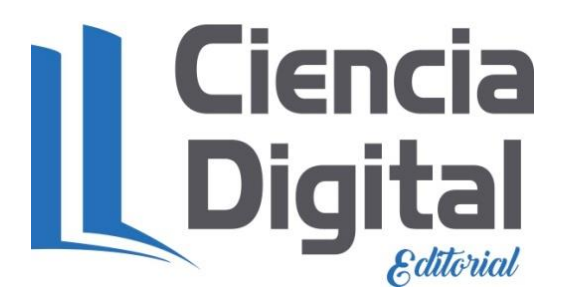


Para citar el artículo indexado.

Velasteguí López, E. (2019). Fomentar Atractivos Turísticos en Guayaquil. ConcienciaDigital, 2(4), 66-77. https://doi.org/10.33262/concienciadigital.v2i4.934

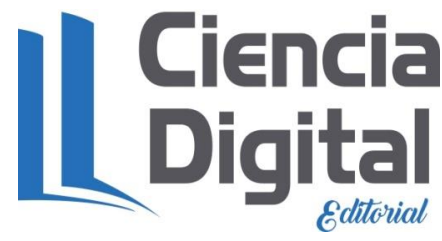

El artículo que se publica es de exclusiva responsabilidad de los autores y no necesariamente reflejan el pensamiento de la Revista Conciencia Digital.

El articulo queda en propiedad de la revista y, por tanto, su publicación parcial y/o total en otro medio tiene que ser autorizado por el director de la Revista Conciencia Digital.

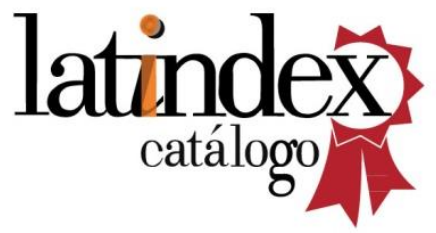

Conciencia

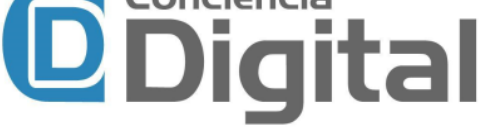

